\title{
Communication
}

\section{Investigating the Usability of a Head-Mounted Display Augmented Reality Device in Elementary School Children}

\author{
Luisa Lauer $^{1, *(\mathbb{D})}$, Kristin Altmeyer ${ }^{2}$, Sarah Malone ${ }^{2} \mathbb{D}$, Michael Barz ${ }^{3,4} \mathbb{D}$, Roland Brünken ${ }^{2}$, Daniel Sonntag ${ }^{3,4}$ \\ and Markus Peschel ${ }^{1}$ (D)
}

check for updates

Citation: Lauer, L.; Altmeyer, K.; Malone, S.; Barz, M.; Brünken, R.; Sonntag, D.; Peschel, M. Investigating the Usability of a Head-Mounted Display Augmented Reality Device in Elementary School Children. Sensors 2021, 21, 6623. https://doi.org/ $10.3390 / \mathrm{s} 21196623$

Academic Editors: Vittorio M.

N. Passaro and Christoph

M. Friedrich

Received: 31 August 2021

Accepted: 30 September 2021

Published: 5 October 2021

Publisher's Note: MDPI stays neutral with regard to jurisdictional claims in published maps and institutional affiliations.

Copyright: (c) 2021 by the authors. Licensee MDPI, Basel, Switzerland. This article is an open access article distributed under the terms and conditions of the Creative Commons Attribution (CC BY) license (https:/ / creativecommons.org/licenses/by/ $4.0 /)$.
1 Department of Physics, Campus C6.3, Saarland University, 66123 Saarbrücken, Germany; markus.peschel@uni-saarland.de

2 Department of Education, Campus A4.2, Saarland University, 66123 Saarbrücken, Germany; kristin.altmeyer@uni-saarland.de (K.A.); s.malone@mx.uni-saarland.de (S.M.); roland.bruenken@uni-saarland.de (R.B.)

3 German Research Center for Artificial Intelligence (DFKI), Interactive Machine Learning Department, Stuhlsatzenhausweg 3, Saarland Informatics Campus D3_2, 66123 Saarbrücken, Germany; michael.barz@dfki.de (M.B.); daniel.sonntag@dfki.de (D.S.)

4 Applied Artificial Intelligence, Oldenburg University, Marie-Curie-Str. 1, 26129 Oldenburg, Germany

* Correspondence: luisa.lauer@uni-saarland.de; Tel.: +49-681-302-71397

\begin{abstract}
Augmenting reality via head-mounted displays (HMD-AR) is an emerging technology in education. The interactivity provided by HMD-AR devices is particularly promising for learning, but presents a challenge to human activity recognition, especially with children. Recent technological advances regarding speech and gesture recognition concerning Microsoft's HoloLens 2 may address this prevailing issue. In a within-subjects study with 47 elementary school children (2nd to 6th grade), we examined the usability of the HoloLens 2 using a standardized tutorial on multimodal interaction in AR. The overall system usability was rated "good". However, several behavioral metrics indicated that specific interaction modes differed in their efficiency. The results are of major importance for the development of learning applications in HMD-AR as they partially deviate from previous findings. In particular, the well-functioning recognition of children's voice commands that we observed represents a novelty. Furthermore, we found different interaction preferences in HMD-AR among the children. We also found the use of HMD-AR to have a positive effect on children's activity-related achievement emotions. Overall, our findings can serve as a basis for determining general requirements, possibilities, and limitations of the implementation of educational HMD-AR environments in elementary school classrooms.
\end{abstract}

Keywords: head-mounted displays; augmented reality; human activity recognition; usability; elementary education

\section{Introduction}

Augmented reality (AR) is an emerging technology in education that enables real-time integration of real and virtual objects in the field of view [1,2]. The real world represents the main channel of perception, and virtual objects are spatially and/or semantically connected to real objects [3]. In educational settings in particular, this offers a great potential to enhance learning processes and, therefore, there is a high interest in the development and research of AR-environments and devices in this context. In particular, head-mounted displays enable an engaging interaction with real and virtual objects. Recent review studies and meta-analyses have confirmed the general benefits of AR-applications for learning [4-6]. However, it is noticeable that children of elementary school age benefit less than older students [7]. The usability of the applied devices seems to play a significant role in the success of AR-applications [8]. Technology for the recognition of user activities and behavior is referred to as 'human activity recognition' (HAR) technology $[9,10]$. It is 
suspected that HAR of AR-devices such as Microsoft's HoloLens (first generation) was not yet technologically mature enough to enable interference-free learning in younger children. However, AR-technology has evolved and may be able to make up for the shortcomings of the past, allowing the potential for AR to be suitable for younger students. Therefore, the current study aimed to test the usability of the HoloLens 2 for elementary school students and to provide an empirical basis to decide whether it is worthwhile to develop school-related learning scenarios for the device. In addition, we examined which of the offered multimodal interaction modes can be handled best by the students.

\subsection{Theoretical and Empirical Background for the Use of HMD-AR in Education}

Following Santos et al. [11], the overlay of the physical world with external representations through AR enables situated multimedia learning. Based on this approach, through AR it is attempted to combine the best of two worlds: situated active learning in a meaningful real-world environment and virtual learning environments carefully designed according to the principles of the Cognitive Theory of Multimedia Learning [12]. Initial studies indicate that through fulfilling the spatial contiguity principle, AR-based learning environments can reduce cognitive load [13,14] and increase learning gains [15]. Moreover, Szajna et al. [16] found that HMD-AR-based applications for training can significantly reduce the time required to perform tasks.

Most educational AR-applications are designed for handheld display-devices like smartphones or tablets, while head-mounted display AR-devices (HMD-AR-devices) are used rarely [4]. Nevertheless, HMD-AR-devices provide several advantages when used in educational settings. In contrast to handheld devices, learners wearing see-through HMD-AR-devices experience a seamless merge of virtual and physical worlds. From the perspective of multimedia learning, this should facilitate the creation of meaningful cognitive relations between virtual information and the physical environment, improving learning outcomes [12,17]. Moreover, unlike handheld display-devices, HMD-AR-devices allow for freehand interaction with physical as well as virtual objects [18]. This becomes particularly useful for learning settings based on physical activities, like laboratory work, which requires leaners to use both of their hands [19]. Theories of embodied cognition suggest that bodily interactions with a learning task, such as hand and finger gestures, can support cognitive processes [20]. Furthermore, Korbach et al. [21] used 2D multimedia learning material to show that using the index finger for pointing and tracing related information influences a learner's focus of visual attention and promoted the learning process. Since HMD-AR-based learning environments enable the presentation or adaptation of learning information based on a learner's gesture or action in real-time, positive effects of embodied cognitions are expected be particularly strong [22].

According to Yuen et al. [23], educational AR-applications can be designed for discoverybased learning (DBL), object modelling (OM), game-based learning (GBL), for the teaching of specific skills in training, or they can be integrated into distinct educational AR-books, with GBL and OM being the most frequently addressed purposes of educational ARapplications [24]. AR in education can help learners to conduct authentic explorations in the real world by displaying virtual elements [25], and can facilitate the observation of processes that cannot be perceived with the naked eye [26]. Further, AR opens new opportunities for the individualization of the learning process through real-time interaction between reality and virtuality as real-time reaction and adaption to the learner's actions [27]. For instance, recent technological advances enable the augmentation of relevant real objects that were fixated by a learner [28]. Besides promoting the acquisition of knowledge and skills [5,29], AR can positively influence curiosity [30] as well as motivation and interest [31] in educational situations. Motivation and interest are known to be modulated by so-called activity emotions, which, as a type of achievement emotions, concern ongoing achievementrelated activities [32,33]. While positive, activating emotions (e.g., enjoyment) are assumed to promote motivation and interest, negative, rather deactivating emotions (e.g., boredom), 
are associated with their decline. Therefore, emotions such as enjoyment, boredom, etc. are referred to as 'activity-related achievement emotions'.

However, the use of AR in education can be obstructed by technical issues and can require additional instruction [34]. Hence, well-designed user interfaces in AR-applications are essential for successful learning [35]. Due to the prevailing research gap concerning the use of HMD-AR-devices and applications in education, as well as ongoing technical advancements concerning HMD-AR-devices, further research is required to validate the existing results and to investigate the effects of HMD-AR on the learning process.

\subsection{Usability of HMD-AR-Devices in Education}

In order for AR-devices to exert their positive impact on information processing during learning, the handling and interaction with the device itself or with the virtual learning information offered must not itself lead to load on the learners as described in the previous Section. According to several reviews on AR in education, an often-reported issue concerning the practical use of AR-devices and applications in educational situations is the underwhelming usability $[4,36,37]$. The (technical) usability of an educational technologysupported setting, which comprises technically conditioned aspects of use and operation, influences the overall usefulness of a learning application [38]. While good usability of educational AR-applications facilitates learning, poor usability can even hamper learning processes [39]. Further, Papakostas et al. [40] found the usability to be the strongest predictor of the behavioral intention to use an AR-application for training. For HMD-AR-devices, a poor performance of the user activity recognition concerning the detection of operation commands can impact usability, as the device is operated through gesture- or voice-based interaction [41]. This aspect is more important when using the devices with young children, as their physical body characteristics (e.g., hand size, arm length, voice pitch) differ from adults [42], for whom the devices are currently designed and calibrated. Previous research concerning the (technical) usability of HMD-AR-devices focused mainly on Microsoft's HoloLens (first generation) and samples of adults. An evaluation of the device for the purpose of an assembly application for manufacturing [18] found the device to be applicable, but also revealed that the spatial mapping required improvement. Munsinger et al. [43] used the Microsoft HoloLens (first generation) to investigate its usability for a target group of elementary school children. They compared three AR-interaction modes provided by HoloLens ('remote clicker', 'air-tap', 'voice command') in their efficiency using the measures 'input errors' 'tutorial time' and 'game time', and found that the 'voice command'-interaction performed significantly worse than the other two. Their findings are in line with rather poor performance occurring for interactive devices with voice-based operation in general $[41,44]$. Besides their physical body characteristics, the children's individual state of cognitive development concerning motoric skills and spatial cognition [42] may affect the usability of HMD-AR-devices.

For many applications, multimodal interfaces have long been recognized to be more robust, accurate, and preferred by users than unimodal ones. A major benefit is that users can freely choose their preferred modality combination [45]. However, this requires the ability to make a good modality choice, because ineffective interaction modalities may lead to unsatisfactory results [46]. Still, multimodal interfaces are considered to be "especially well-suited for applications like education, which involve higher levels of load associated with mastering new content" [47] (p. 33). As the HoloLens 2 offers different means to multimodally interact in AR, we investigate the preferred modality choices of elementary school children. So far, investigations on children's handling with the revised interaction modes of the latest HoloLens 2 are still pending.

\subsection{The Microsoft HoloLens 2 and Its Potential for Education}

Announced innovations and improvements concerning the spatial positioning, speech, and gesture recognition for the successor model HoloLens 2 by Microsoft (see Figure 1) do not only make it necessary to investigate the applicability of existing findings for the new 
device. The new device could further represent an important step towards user-friendly HMD-AR-applications for educational purposes, especially for young children.

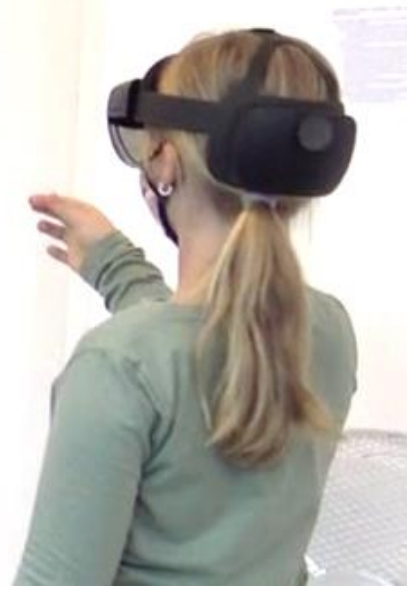

(a)

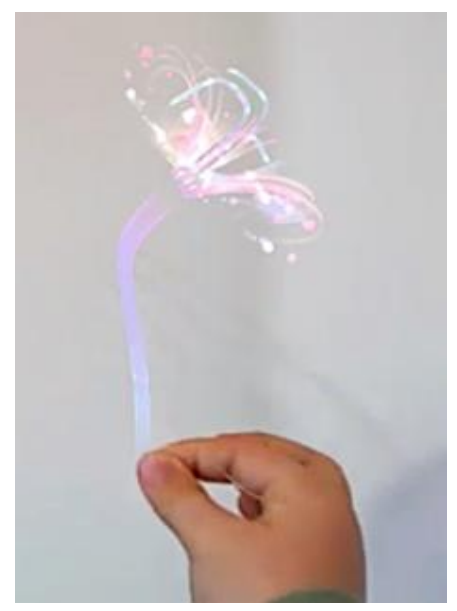

(b)

Figure 1. Child interacting with a virtual object in AR using Microsoft's HoloLens 2. (a) external view; (b) child's point of view.

The HoloLens 2 offers various means to interact in AR. To describe these modes of interactions, we will focus on the action 'selection of an AR-object' from the tutorial that is pre-installed on the device (see Video S1a). The AR-object to select in the tutorial was a shimmering gemstone. On the one hand, there are gesture-based interactions: To select the AR-gemstone with a gesture-based interaction, one can either tap directly on the gemstone (newly implemented 'tap'-interaction, see Figure 2a and Video S1a) or one can aim at the gemstone from a distance with the open palm and then tap with the thumb and index finger ('air-tap'-interaction, see Figure $2 \mathrm{~b}$ and Video S1a). On the other hand, there is voice-and-gaze-based interaction: To select the AR-gemstone, one can also look at the gemstone and say 'select' ('voice command'-interaction, see Figure 2c and Video S1a). In total, two gesture-based and one voice-and-gaze-based interaction mode are available for AR-interaction on the HoloLens 2.

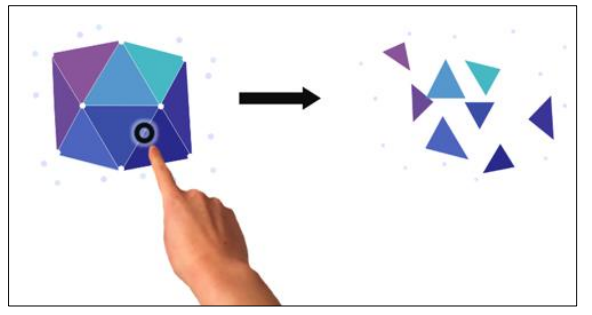

(a)

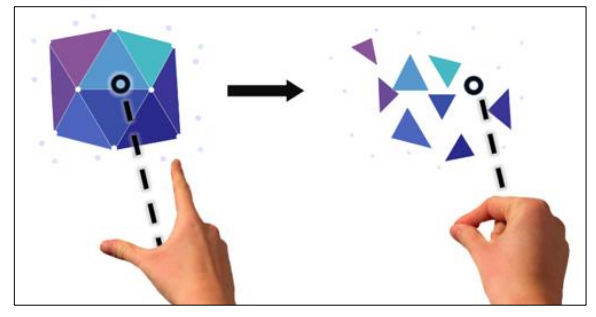

(b)

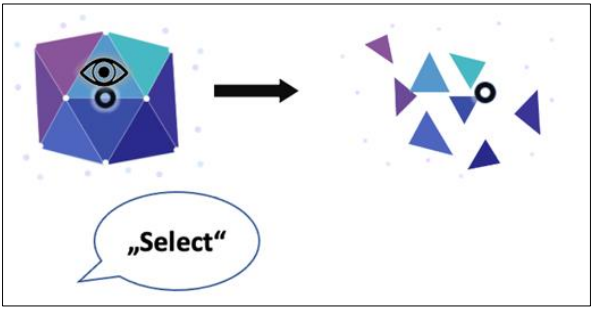

(c)

Figure 2. AR-interaction modes provided by HoloLens 2. (a) 'tap'; (b) 'air-tap'; (c) 'voice command'.

\subsection{Ethics of Using HMD-AR Devices in Elementary Education}

Children are vulnerable and it is the responsibility of adults to protect them from possible harm. Technologies in research on children should therefore be applied very prudently. Particularly when using immersive technologies, such as AR and virtual reality (VR), special precautions should be taken [48]. To ensure that the psychological and cognitive state of the target group was taken into account, our research team included experts in the fields of infant mental development (psychologists) and elementary school pedagogy (teachers and researchers). These considerations led us to gently introduce the 
children to the technology: we first showed them the device and explained how it works in a child-friendly way. While they were using the smartglasses, an experimental supervisor was always on hand to help them. We also monitored the physical well-being of the children [49] by asking them repeatedly whether they experienced any discomfort in terms of simulator sickness. Moreover, the virtual content of the AR environment used does not contain frightening or startling elements. To protect the children's data (e.g., eye movement recordings [50]), we used a private offline Wi-Fi to enable Mixed Reality Capture.

\subsection{Aim of the Study}

Our aim is to assess the usability of Microsoft's HoloLens 2 as the latest HMD-ARdevice for the use with elementary school children. The device is not yet technically designed for use with children younger than 13 years: young children's lower interpupillary distance might hamper the perception of virtual objects [51]. Therefore, we want to explore how usable the device is in its current state, and which technical adaptions need to be carried out before the device can be successfully used with young children, following similar evaluations for the predecessor model by Munsinger et al. [43]. We want to gain an insight into the general challenges and benefits that can serve as baseline findings once the device is used in educational applications. Our main research focuses concerning the use of the device are:

1. Evaluation of the overall usability of the HoloLens 2 as an HMD-AR-device;

2. Comparison of the provided AR-interaction modes concerning their efficiency;

3. Assessment of the children's interaction preference in HMD-AR;

4. Examination of the change in activity-related achievement emotions.

\section{Materials and Methods}

\subsection{Sample}

We invited 47 students ( $29 \%$ female, age: $M=9.3$ years; $S D=0.9$ years, 2 nd to 6th grade) to participate in a laboratory study at the Saarland University. They took part in another study at the same day (either before or after attending this study), but the other study did not include the use of an HMD-AR-device. None of the children had previous experience with AR. In the beginning, we conducted test runs with four children (for procedure and instruction refinement, without data collection), so $n=43$ valid data sets were collected.

\subsection{Study Design}

The study was conducted using a within-subjects design. The independent variable was interaction mode, and the modes were modeled as different measuring points. The different multimodal AR-interaction modes provided by HoloLens 2 ('tap', 'air-tap', and 'voice command') were compared regarding the dependent variables 'mean number of attempts' and 'mean time'. For the children's personal interaction preference in AR, we formed the variables 'most favorite interaction mode' and 'least favorite interaction mode'. The most and the least favorite interaction mode can be 'tap', 'air-tap' or 'voice command'. To investigate general effects of HMD-AR-usage on activity-related achievement emotions, we formed a pre- and a post-test variable for 'enjoyment', 'boredom' and 'frustration'. To assess the overall device usability, we formed the variable 'system usability score'.

\subsection{Procedure and Data Collection}

Due to the COVID-19 situation, only individual appointments with private journeys could be made. Prior to the start of the study, parents were informed about the investigation and gave their written consent for their children's study participation. The procedure of the study was centered around a standardized tutorial on interaction in HMD-AR on the HoloLens 2 in German language. Before starting the tutorial, we assessed the children's enjoyment, boredom and frustration. These activity-related achievement emotions are assumed to allow for inferences about motivation and interest [32,33] (variables 'enjoyment- 
pre', frustration-pre', 'boredom-pre'). Each emotion was assessed using a single item adapted for children from Riemer and Schrader [52] (see Appendix A and Document S1b). Moreover, children were asked about their previous experience with AR. The children were then introduced to the HoloLens 2 and the concept of HMD-AR by showing them the 'Mixed Reality Capture' (livestream) while the experimental supervisor was wearing the device (see Figure 3). We thoroughly instructed the children to handle the device carefully and explained that it is not a toy. Afterwards, the experimenter mounted the device on the child's head and an eye calibration was carried out. As described in Section 1.5, the device is currently designed for adults and the manual states that children under the age of 13 years might not be able to see virtual objects comfortably due to a low interpupillary distance. We therefore asked the children after the eye calibration whether they had any problems in seeing the virtual objects, especially reading texts. Then, the children were informed that they were going to learn different methods of interaction in AR and went through the standardized tutorial on multimodal interaction in HMD-AR that is pre-installed on the HoloLens 2 (see Video S1a). The tutorial includes several interaction scenarios. For our analysis, we focus on the task 'selecting a gemstone' only because it is available for all interaction modes. The task 'selecting a gemstone' is the first shown in the tutorial. The three interaction modes are introduced one after the other and the order of the tutorial tasks is fixed ('tap'-'air-tap'-'voice command'). At the beginning, three gems are shown. They must be selected one after the other with the respective method. During the entire tutorial, the gems can only be selected with the interaction method that is currently being introduced. An invisible speech-based virtual agent explains what to do in each case, and this information is additionally displayed in text form. For 'tap' the translated instruction is: "Tap a nearby gem with your finger to select it." The translated instruction for 'air-tap' is: "Aim the beam from your palm at holograms out of range. Tap to select with your index finger and thumb and release." For the 'voice-command'-interaction, the translated instruction is: "Target a gem with the gaze cursor and say 'Select'). The auditory explanation is played only once, while the text remains visible. If the correct (gesture or voice) input does not follow immediately, help is given depending on the interaction method: For gesture-based interaction, a hand appears that repeats the correct gesture until the gem is successfully selected. In voice-based interaction, the text "say <Select>" appears when a gemstone is targeted with the gaze cursor. However, the tutorial behaves the same way for an incorrect input (e.g., an incorrect gesture or voice command) and there is no feedback reporting that the input is incorrect. Therefore, if the child had a difficulty in understanding the instruction, the experimental supervisor helped by repeating or explaining the instruction given via voice and text. A successful selection is visually indicated by the vanishing of the selected gemstone and a short audio signal. Once all three gemstones are successfully selected, the tutorial automatically proceeds to the next task. To assess the efficiency of the different AR-interaction modes, we asked the children to perform each interaction mode for selecting a gemstone three times. We counted the number of attempts (to calculate the variable 'mean number of attempts') and the time (to calculate the variable 'mean time') for each of the three tries for 'tap', 'air-tap' and 'voice command'. The tutorial performance was recorded with an external camera and with the POV-camera from the HoloLens 2 (see Figure 3) to validate the data for the two variables and to document possible technical issues that may influence the usability. After the tutorial, we asked the children to express their current activity-related achievement emotions with another questionnaire. They had to state their enjoyment, boredom and frustration in comparison to the previous questioning (variables 'enjoyment-post', 'frustration-post, 'boredom-post') (see Document S1b). Pilot studies in which the questionnaires were developed had shown that children were very joyfully excited when they came to the experiment and therefore rated positive emotions as high as possible on the scale and negative emotions as low as possible on the scales. Adjustments were needed to allow the children to express that their positive emotions were even more positive or negative emotions were even less than before interacting with the AR-device. In addition, the children rated the overall system usability 
(variable 'system usability score') of the HoloLens 2 as an HMD-AR-device. The used System Usability Scale (SUS) [53] comprises ten statements on different facets of system usability. The statements were translated into German and the wording was simplified to match the target group of children (see Appendix B and Document S1c). To assess the children's preference concerning the interaction modes provided by the device, we showed them schematic pictures of the 'tap'-, 'air-tap'- and 'voice command'-interactions and re-explained the interaction modes to them after completing the tutorial. The children were asked to rank the interaction modes based on their personal preference (variables 'most favorite interaction mode' and 'least favorite interaction mode') and to provide explanations for their decisions. Finally, we asked the children to report any perceived inconveniences, e.g., pain from wearing the device or problems seeing objects or reading text in AR and to report any inconveniences that are related to simulator sickness [54].

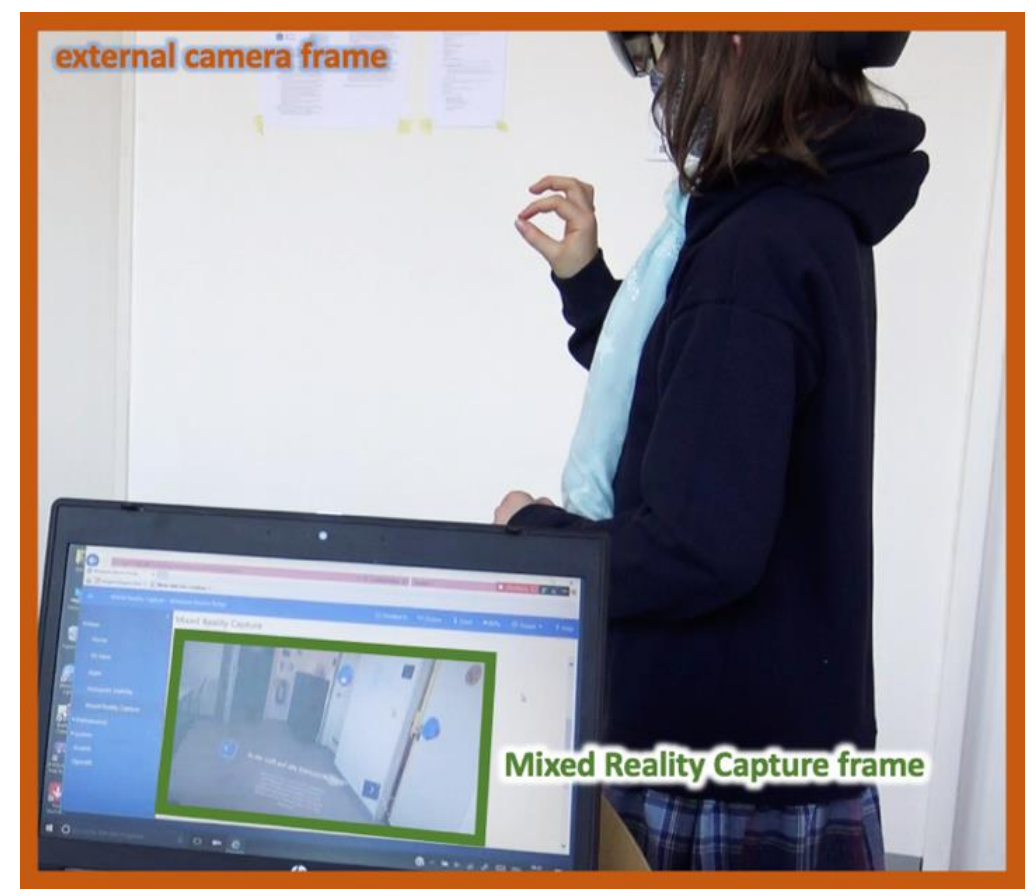

Figure 3. Video recording during the tutorial: An external camera and the 'Mixed Reality Capture' accessible through Microsoft Device Portal (via Browser) were used.

\subsection{Data Analysis}

First, we calculated the SU score. Subsequently, we compared the interaction modes with respect to their efficiency and evaluated the children's interaction preferences in AR. Lastly, we examined the changes in the activity-related achievement emotions.

\subsubsection{Overall Device Usability}

The overall system usability was assessed using the variable 'system usability score'. It is calculated as a descriptive measure from the children's answers to the SUS-questionnaire. We used a five-point instead of the original ten-point-Likert scale to ease the rating process for the children and adjusted the score calculation accordingly. The scale is acceptably reliable (Cronbach's Alpha $=0.76$ ) for the sample. In addition, technical issues taken from the recorded tutorial videos that may impact the usability when wearing or using the device are described. 


\subsubsection{Efficiency of the AR-Interaction Modes}

The dependent variables 'mean number of attempts' and 'mean time' are compared for the three measurement points 'tap', 'air-tap' and 'voice command' using two respective non-parametric Friedman-tests for differences among repeated measures.

\subsubsection{Interaction Preference in AR}

The quantitative distributions of the children's most and least favorite interaction mode in AR are reported. Reasons given for their decisions are presented.

\subsubsection{Changes in Activity-Related Achievement Emotions}

Changes regarding the activity-related achievement emotions were assessed by three Wilcoxon-signed-rank-tests for paired samples comparing the pre and posttest variables 'enjoyment', 'boredom' and 'frustration'. Each emotion item of the pretest is based on a fivepoint Likert-scale (options: 'totally disagree' - 'rather disagree' - 'neither' - 'rather agree' 'totally agree'). In the second questionnaire the children rated their current emotions in comparison to the previous measuring point (options: 'much less' - 'a little less' 'unchanged'-'a little more'-'much more'). Therefore, data transformation was applied. For further details see Appendix A.

\section{Results}

\subsection{Overall Device Usability}

The 'system usability score' was calculated based on the children's answers on the five-point Likert-scale with high ratings on regular items increasing the score and high ratings on inverse items decreasing the score following Brooke [53] (see Document S1c). The median system usability score is 80 (maximum: 100) which indicates a 'good' system usability [55].

Apart from minor (situational) technical issues, one (that may impact the device usability) occurred frequently: In 19 out of 43 subjects (56\%), the AR-objects moved away from the child's hand as it approached the object. This occurred when the child had to move the body towards an object to reach it, but not when the child stood still when interacting with an object. For a demonstration of the issue see Video S1d. Furthermore, none of the subjects reported any physical inconveniences related to simulator sickness.

\subsection{Efficiency of the AR-Interaction Modes}

The two non-parametric Friedman-tests of differences among repeated measures (for descriptive statistics see Table 1) revealed significant differences among the three interaction modes 'tap', 'air-tap' and 'voice command' concerning the required number of attempts (Chi-Square $(2)=72.29, p<0.001)$ and the required time (Chi-Square $(2)=82.19$, $p<0.001$ ). Pairwise post-hoc comparisons (see Table 2) indicate that the 'air-tap'-interaction requires more attempts compared to 'tap' and 'voice command'. Concerning the required time, all three interaction modes differ significantly, with 'tap' being the fastest and 'air-tap' requiring the most time to perform. To conclude, the 'tap'-interaction appears to be the most efficient for both interaction preferences. The 'voice command'-interaction appears to be the second-best interaction mode. The 'air-tap'-interaction is noticeably less efficient to use. 
Table 1. Descriptive statistics for the efficiency assessment.

\begin{tabular}{cc}
\hline Dependent Variable & Mean (SD) \\
\hline mean number of attempts for 'tap' & $1.001(0.508)$ \\
mean number of attempts for 'air-tap' & $2.763(1.549)$ \\
mean number of attempts for 'voice command' & $1.194(0.771)$ \\
\hline mean time [s] for 'tap' & $1.200(0.346)$ \\
mean time [s] for 'air-tap' & $16.047(13.443)$ \\
mean time [s] for 'voice command' & $3.672(6.007)$ \\
\hline
\end{tabular}

Table 2. Pairwise Dunn-Bonferroni post-hoc comparisons for the efficiency assessment.

\begin{tabular}{ccccc}
\hline $\begin{array}{c}\text { Dependent } \\
\text { Variable }\end{array}$ & $\begin{array}{c}\text { Compared Interaction } \\
\text { Modes }\end{array}$ & $\mathbf{Z}$ & $\begin{array}{c}\boldsymbol{p} \\
\text { (Two-Tailed) }\end{array}$ & $\begin{array}{c}\boldsymbol{r} \\
\text { (Cohen) }\end{array}$ \\
\hline \multirow{2}{*}{$\begin{array}{c}\text { mean number of } \\
\text { attempts }\end{array}$} & tap-air-tap & -1.49 & $<0.001^{* * *}$ & 0.227 \\
& tap-voice command & 0.22 & 0.306 & \\
& air-tap-voice command & 1.27 & $<0.001^{* * *}$ & 0.193 \\
\hline \multirow{2}{*}{ mean time } & tap-air-tap & -1.95 & $<0.001^{* * *}$ & 0.298 \\
& tap-voice command & -1.047 & $<0.001^{* * *}$ & 0.215 \\
& air-tap-voice command & 0.907 & $<0.001^{* * *}$ & 0.138 \\
\hline
\end{tabular}

Significance levels: ${ }^{* * *} p<0.001$.

\subsection{Interaction Preferences in $A R$}

As shown in Figure 4, most children preferred the 'voice command'-interaction. 'Tap' and 'air-tap' were chosen equally frequent. The most often stated reason for the choice of preference was the simplicity of interaction in AR (for 'tap' and 'voice command'). The children who favored the 'air-tap'-interaction mostly argued that it was fun to use. However, almost 60 percent of the children stated 'air-tap' as their least favorite interaction mode, arguing that it was difficult to use. 'Tap' and 'voice command' were chosen almost equally frequent for various reasons.

\section{Most favorite interaction mode}

\begin{tabular}{|c|c|c|}
\hline 12 (28 \%) & $12(28 \%)$ & 19 (44\%) \\
\hline simple (12) & $\begin{array}{l}\text { fun (8) } \\
\text { usable from afar ( } 3 \text { ) } \\
\text { challenging (1) }\end{array}$ & $\begin{array}{l}\text { simple (17) } \\
\text { fun (1) } \\
\text { usable from afar (1) }\end{array}$ \\
\hline
\end{tabular}

\section{Least favorite interaction mode}

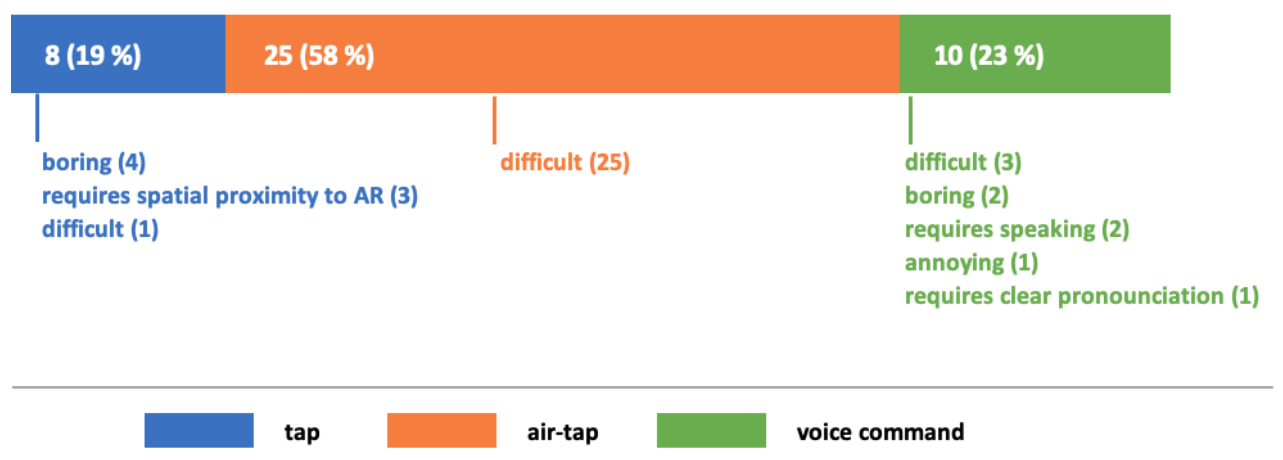

Figure 4. Distributions for most and least favorite interaction mode in AR with reasons for decisions ( $\mathrm{N}=43$ ). 


\subsection{Changes in Acitivity-Related Achievement Emotions}

The Wilcoxon-signed-rank-tests for paired samples (see Table 3) revealed an increase in enjoyment $(Z=-5.641, p<0.001)$, and a decrease in boredom $(Z=-5.031, p<0.001)$ and frustration $(Z=-5.097, p<0.001)$.

Table 3. Descriptive statistics and signs (Wilcoxon-signed-rank-test) for the for the activity emotion change assessment.

\begin{tabular}{ccccc}
\hline Dependent Variable & Mean ${ }^{\mathbf{1}}(\mathrm{SD})$ & Pos. Signs & Neg. Signs & Ties \\
\hline enjoyment-pre & $6.350(0.613)$ & 36 & 0 & 7 \\
enjoyment-post & $7.880(0.981)$ & & 30 & 11 \\
boredom-pre & $3.370(0.817)$ & 2 & & \\
boredom-post & $2.090(1.250)$ & & 27 & 15 \\
frustration-pre & $3.050(0.213)$ & 1 & \\
frustration-post & $1.840(1.022)$ &
\end{tabular}

${ }^{1}$ Range for transformed means: 1(very low)—9(very high).

\section{Discussion}

To sum up, HoloLens 2 has proven to be an effective and appropriate HMD-AR-device to use with elementary school children, also confirmed by their subjective usability ratings. While children managed to use all provided interaction modes, the newly implemented direct 'tap'-interaction turned out to be the best performing mode of interaction with virtual elements. Their reported most favorite interaction mode, however, was the 'voicecommand'-interaction. Although children were unfamiliar with HMD-AR-devices, the positive effects on activity-related achievement emotions suggest that educational settings may even benefit from using the HoloLens 2.

\subsection{Overall Device Usability}

The usability of the device was rated with an average system usability score of 80 , resulting in the rating 'good' (scores greater than 82 indicate "excellent" usability) [55]. Still, further improvements are required for an effective application of the HoloLens 2 in educational applications because any usability flaw can hamper the learning performance (see Section 1.2). This can be achieved through, e.g., further technical improvements or specific tutorials adapted to the respective application and target group. As AR-technology and AR interactions were largely unknown to the children, it is possible that the extensive instruction required before and during the tutorial could have had a negative impact on the system usability score.

The frequently observed technical issue of AR objects moving away from children could stem from the device settings concerning the relative positioning of AR objects to the viewer, which seem to be designed for adults and their body dimensions. This did not occur in any of the adults that used the device. However, this is a preliminary statement without statistical validation as we did not investigate the occurrence of this issue comparing children with adults. It is assumed that the spatial position of the AR-objects in the tutorial is preset for adults and their physical appearance and that the children need to make a step towards an object to reach it. Yet, this causes the AR-objects to move away from the child as the device registers the spatial movement of the child and tries to maintain the relative position between spectator and AR-object. For a successful use of the device in educational situations with young children, the relative positioning of AR-objects should therefore either be scaled down to the length of children's arms or absolute object positioning should be selected instead.

The absence of physical inconveniences related to simulator sickness might be explained by the fact that the familiar real world remains visible in AR. Our results are consistent with findings for the HoloLens (first generation) [56], that revealed the device to only cause negligible symptoms of simulator sickness in training. Simulator sickness 
has so far been observed more frequently in VR experiences [57], in which the subjects are visually isolated from their reference world.

\subsection{Efficiency of the AR-Interaction Modes}

As described in Section 3.2, we found the 'tap'-interaction to be most efficient concerning our applied measures (mean task attempts, mean task time) without prior training for the children, as it requires a low number of attempts and can be performed quickly. The 'voice command'-interaction was found to be the second-best interaction mode (low number of attempts, but higher required performance time). The 'air-tap' mode is the least efficient to use, as it requires both a high number of attempts and a long time to perform. Based on our experience during the data collection, this might be since the 'airtap'-interaction is only registered successfully by the device if the object is selected by the hand beam long enough and if the 'tap' gesture is performed clearly. It is possible that the children's physical and motoric body characteristics [42] additionally complicated the detection of the gesture.

As we found the mean task time to be significantly higher for 'voice command' in comparison to 'tap', which we assume is caused by the longer action processing time of the voice-and-gaze-based interaction. It is necessary for the person to say the command, and for the device to detect and process it, while for the gesture-based interactions, the device can make use of the real-time gesture tracking. In their study with the predecessor model (HoloLens first generation), Munsinger et al. [43] found the 'voice command'interaction to produce more input errors than the 'air-tap'-interaction. They further found the 'voice command' to require more time to perform than the 'clicker' (which is not available for the HoloLens 2), but not more time than the 'air-tap'-interaction. Our results deviate from these findings as we found the 'voice command'-interaction of the latest HoloLens to be noticeably improved and generally efficient and to not be overall inferior to the best performing interaction mode (i.e., the direct 'tap'-interaction, which was not available on the HoloLens first generation). This improvement was evident even though the children in this study wore medical face masks, which may have limited the clarity of their pronunciation. However, we cannot accurately compare our results concerning the pairwise comparisons between the AR-interaction mode, as we used different study designs (within-subjects design vs. experimental design) and different procedures (focus on the first three attempts with an interaction mode vs. observation after a familiarizing phase).

In our model, we assume that a higher required number of attempts indicates a lower efficiency. However, especially for gesture-based AR-interaction, it could be more efficient to perform several attempts in a short period of time than to only make one attempt. On the other hand, the opposite might be the case for voice-and-gaze-based interaction in AR, based on our experience. Consequently, we did not compute a single efficiency measure from the two measures used in this study, as they could scale in different directions in terms of efficiency. Therefore, the interpretation of the calculated measures regarding the efficiency of the methods must be done cautiously. The efficiency effect of performing several gesture-attempts in a shorter period appeared to be evident for the 'air-tap'-interaction, which is the more complex gesture-based AR-interaction mode in this study. This effect might even be more prominent in more complex gesture-based interactions like the rotation of an object. However, the measure 'mean time' appears to be overall applicable for interpretation concerning efficiency, as a lower completion time means higher efficiency for any AR-interaction mode.

\subsection{Interaction Preferences in $A R$}

We found that the children's favorite mode to interact in AR was the 'voice command'. However, 'tap' and 'air-tap' were chosen equally often. We therefore conclude that the preferences concerning AR-interaction vary among the children. For 'tap' and 'voice command', the most given reason for the stated preference was the perceived simplicity of the task. This indicates that the children made their choice based on their personal 
experience with the respective AR-interaction modes. Yet, it appears that they did not necessarily make their choice based on the efficiency measures that we applied to compare the AR-interaction modes since the 'voice-command'-interaction was chosen most often It could be the case that the children who favored the 'voice-command'-interaction found it easier to say the command than to tap at the object, despite performing 'more efficient' with the 'tap'-interaction. The children that favored the 'air-tap'-interaction mostly argued that it was fun to use. It appears that this choice was not made based on efficiency, but rather on personal enjoyment. However, the 'air-tap'-interaction appears to be the least favored in general as almost 60 percent of the children claimed to not like it because of its difficulty to perform. This is consistent with our conclusion that it is the least efficient mean to interact in AR.

\subsection{Activity-Related Achievement Emotions}

With the aim of evaluating the applicability of the HoloLens 2 for a target group of children in educational settings, we also analyzed the influence of the HMD-AR-device on activity- related achievement emotions. Results are consistent with prior research [31] and indicate a positive effect of the AR-device on enjoyment, which is assumed to promote motivation and interest [32,33]. Although all children used the innovative and technically challenging AR-device for the first time, results showed a decrease in frustration and boredom, being another hint for its motivational effects. While these promising results on emotions point at possible learning benefits for HMD-AR-supported learning environments, it remains unclear whether the effects persist over time as the children become used to the device and the AR-experience. Moreover, it cannot be assumed that positive emotions concerning learning materials or specific devices improve learning outcomes per see [58]. According to the Cognitive Affective Theory of Multimedia Learning (CATML [59]), emotions impact cognitive processes during multimedia learning, as they affect the learners' engagement. The processes involved are complex. It has been demonstrated, for example, that too much positive emotion can impede learning, and that in some cases even negative emotions can be useful [60]. Thus, when devices that trigger strong emotions are used for learning, it is important to carefully examine what effect this has on learning. Therefore, future studies should address affective processes in learning with the HoloLens 2.

\subsection{General Limitations of the Study}

Due to the necessity to make individual appointments in a laboratory at a university, the sample could have a high proportion of children who are rather interested in AR (or technology in general) as they participated in the study on a voluntary basis and were not invited as a school class. The likely very high proportion of children in the sample with a strong interest in AR could reduce the transferability of the results to the overall population. Therefore, further research with other samples is generally needed to validate or complement the results.

Moreover, the order of the AR-interaction mode presentation in the tutorial was not randomized. The lack of randomization may have produced carry-over effects from the first measuring point ('tap') to the second ('air-tap') and to the third ('voice command'). However, this carry-over could have been diminished by the difference in execution between the presented AR-interaction modes, thus not offering many possibilities to learn something from a task that could be used when performing the next. In an experimental design with split groups for each interaction mode however, we would not have been able to assess the children's personal interaction preference in AR in such semantic proximity to the actual tasks that they experienced as in the within-design that we chose.

Due to the lack of an underlying learning content in the study, the findings cannot be directly transferred into educational situations, and they should be validated or complemented in further studies using multiple activities and learning contents as examples. Nevertheless, the present results can serve as a general basis for the development of any educational HMD-AR application. 
Concerning the lack of technical adaption of the device to the physical body characteristics of young children (especially the lower interpupillary distance), we found that although the device was not purposely developed for children, it proved to be usable. In our study, none of the children reported problems seeing virtual objects (neither when asked right after the eye calibration at the start of the study nor after the tutorial when we asked them to report symptoms related to simulator sickness, including problems with text reading or image perception in AR).

\subsection{Practical Application}

Research findings pointing to the general benefits of $\mathrm{AR}$ in educational contexts, and the present study demonstrating that HMD-AR has the potential to be used with younger learners, suggest that in the future, HMD-AR could be profitably used in classrooms. However, from an economic point of view, smart glasses are very expensive devices and if they are purchased in large numbers, e.g., for a complete course, they represent a high financial outlay for schools. In addition, there may be high software development costs, which are necessary because there are still hardly any purchasable software products for curricular-relevant contents. Therefore, schools and other educational institutions are well advised to implement AR in the classroom only if it has been proven that a specific learning content is better conveyed through AR than via traditional or less expensive media and methods. For learning content in which manual interaction with real or virtual objects is not central to learning success, one can also fall back on the more affordable tablet variant.

\section{Conclusions}

Our study indicates a good system usability of Microsoft's HoloLens 2 when used by elementary school children. The newly implemented direct 'tap'-interaction in AR appears to be most effective without prior training. Despite requiring more time to perform, the 'voice command'-interaction was found to work well with children's voices. This deviates from previous findings for the HoloLens (first generation) and other technologies. Further, we found different interaction preferences in AR among the children in accordance with prior research. Yet, the children's preferences do not seem to be based on objective efficiency. Our study suggests that the HoloLens 2 (as an HMD-AR-device) is generally effective for applications with young children as a target group. However, the provided AR-interaction modes appear to differ in their efficiency, at least during the time of familiarization with the device. We propose that future HMD-AR applications for education offer multiple interaction modes to serve the different interaction preferences in HMD among the children.

Although the research results presented are not based on a specific learning content, they still provide an important point of reference for developers designing HMD-AR based learning applications. However, the particular integration of HMD-AR in educational situations has to be aligned with applied instructional methods and current learning goals. Prior research on HMD-VR-based lessons showed that learning outcomes vary depending on the use of a learning strategy [61]. Nevertheless, the detachment from a learning content allows our findings to be used as a basis to determine general requirements, possibilities, and limitations of the development and implementation of any kind of educational HMD-AR-environments for children. Although our study suggests that the device can be successfully used with elementary school children, a technical adaption concerning the physical body characteristics (e.g., the adaption to lower interpupillary distance) needs to be carried out. Furthermore, more research is needed to verify and complement our findings, as the evaluated device and its associated technologies are still a novelty, with little directly related research available in general. However, due to the continuous technical development of HMD-AR devices, our research only describes the current status and must be revised with the appearance of a successor model or another, better device. Future research in this area could assess the usability of the device in educational settings and compare the usability of the provided AR-interaction modes for actions of higher complexity, e.g., rotating an AR-object or altering its size. 
Supplementary Materials: The following will be made available online at https:/ /www.mdpi.com/ article/10.3390/s21196623/s1, Video S1a: tutorial demo with selected samples, Document S1b: activity emotion questionnaire, Document S1c: system usability questionnaire, Video S1d: spatial repositioning issue demonstration.

Author Contributions: Conceptualization, L.L., S.M. and K.A.; methodology, L.L.; formal analysis, L.L., M.B., S.M. and K.A.; investigation, L.L. and K.A.; resources, M.P., R.B. and M.P.; data curation, L.L.; writing-original draft preparation, L.L., M.B., K.A. and S.M.; writing-review and editing, S.M., M.P., R.B. and D.S.; visualization, L.L.; supervision, S.M., M.P., R.B. and D.S.; project administration, R.B., M.P. and D.S.; funding acquisition, S.M., R.B., D.S., M.P. All authors have read and agreed to the published version of the manuscript.

Funding: This research was funded by Bundesministerium für Bildung und Forschung, grant numbers 01JD1811A and 01JD1811C. We acknowledge support by the Deutsche Forschungsgemeinschaft (DFG, German Research Foundation) and Saarland University within the funding programme Open Access Publishing.

Institutional Review Board Statement: The study was conducted according to the guidelines of the Declaration of Helsinki. The institutional review approval was waived for this study for the following reasons: The participants used a technical device that is approved for private use and performed a preset tutorial. The participants did not have to disclose private personal experiences, sensitive information or attitudes. Participants were not intentionally misinformed or involved in covert observation in a non-public place.

Informed Consent Statement: Written informed consent was obtained from all subjects involved in the study.

Data Availability Statement: The data presented in this study are openly available in Open Science Framework (OSF) via: https: / / mfr.osf.io/render?url=https \%3A\%2F\%2Fosf.io\%2Fdf6w9\%2Fdownload (31 August 2021). For reasons of data protection, the video recordings are not publicly accessible.

Acknowledgments: We would like to thank the anonymous reviewers for their contributions and suggestions to the draft of this paper.

Conflicts of Interest: The authors declare no conflict of interest. The presented research is neither sponsored by nor affiliated with Microsoft Corporation. Microsoft Corporation had no role in the design of the study, in the collection, analyses, or interpretation of data, in the writing of the manuscript, or in the decision to publish the results.

\section{Appendix A}

The children's activity-related achievement emotions were assessed before and after the tutorial on interaction in HMD-AR using the pre-questionnaire comprises three items adapted for children from Riemer and Schrader [52] (see Figure A1). As explained in Section 2.3., pre-studies for the development of the questionnaires had shown that the children rated positive emotions as high as possible on the scale and negative emotions as low as possible on the questionnaire before and after the tutorial. We therefore modified the post-questionnaire to allow children to express their emotions in relation to the previous questioning (see Figure A2).

As described in Section 2.4.4, data transformation was required for the activity emotion assessment. Both questionnaires are based on a five-point Likert-scale, but the second questionnaire asks the children to rate their sentiments in comparison to the previous questioning. To take this comparison into consideration, we transformed the dataset from the first questionnaire from a range of 1-5 to 3-7, and for the second questionnaire, we either increased or reduced the previous value based on the chosen answer on the items as shown in Table A1. 
Table A1. Data transformation for activity-related achievement emotions assessment.

\begin{tabular}{|c|c|c|c|c|c|}
\hline \multicolumn{6}{|c|}{ Pre-test item, e.g., 'I am having fun right now.' } \\
\hline Answer & $\begin{array}{l}\text { 'Totally } \\
\text { disagree' }\end{array}$ & $\begin{array}{l}\text { 'Rather } \\
\text { disagree' }\end{array}$ & ‘Neither' & 'Rather agree' & 'Totally agree' \\
\hline Pre-Score X & 3 & 4 & 5 & 6 & 7 \\
\hline \multicolumn{6}{|c|}{ Post-test item, e.g., 'How much fun you are having right now in comparison to before?' } \\
\hline $\begin{array}{c}\text { Answer } \\
\text { Post-Score Y }\end{array}$ & $\begin{array}{c}\text { 'Much less' } \\
X-2\end{array}$ & $\begin{array}{c}\text { 'A little less' } \\
\text { X }-1\end{array}$ & $\begin{array}{c}\text { 'Unchanged' } \\
X\end{array}$ & $\begin{array}{c}\text { 'A little more' } \\
X+1\end{array}$ & $\begin{array}{c}\text { 'Much more' } \\
X+2\end{array}$ \\
\hline
\end{tabular}

\section{How are you feeling right now? For each statement, please mark what applies best to you!}

\begin{tabular}{|l|}
\hline I am having fun right now. \\
$\begin{array}{c}\text { I think that this is I think that this is } \\
\text { not true at all. } \\
\text { rather not true. }\end{array}$ \\
decide. I cannot I think that this is I think that this is \\
rather true.
\end{tabular}
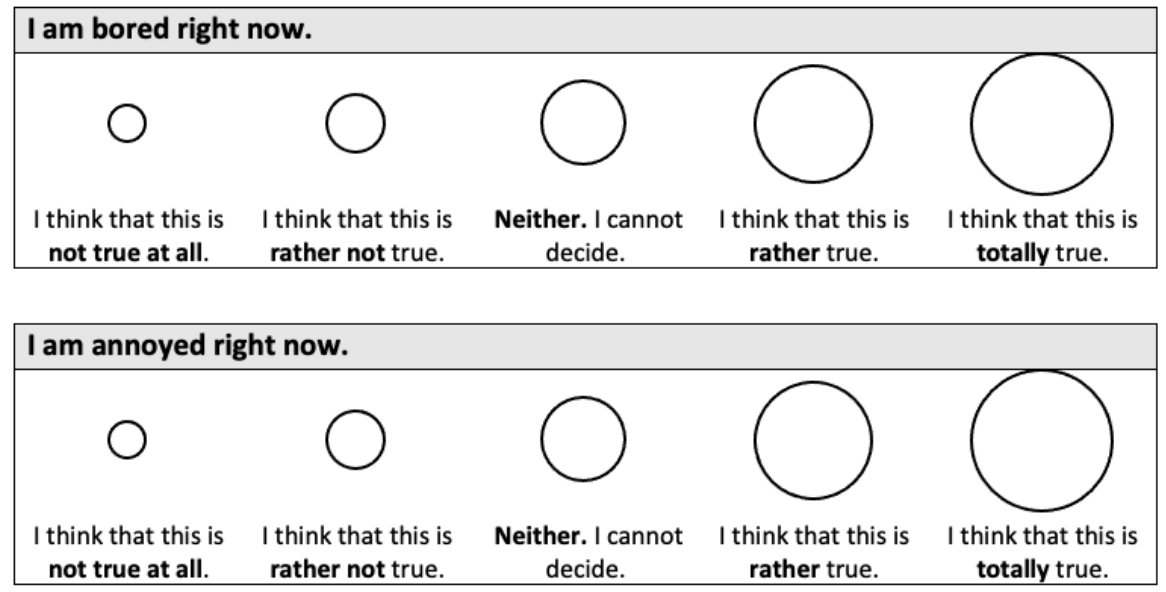

Figure A1. Pre-questionnaire for activity-related achievement emotions assessment.
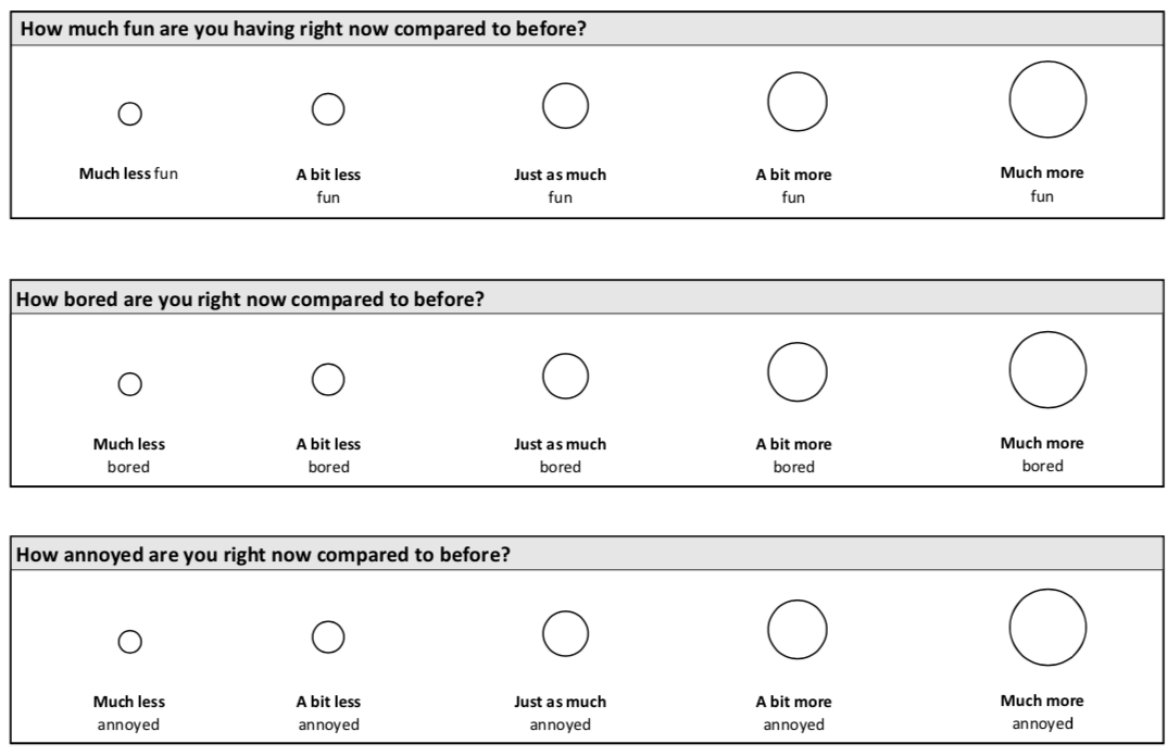

Figure A2. Post-questionnaire for activity-related achievement emotions assessment. 


\section{Appendix B}

The questionnaire for the overall device usability assessment described in Section 2.3. consists of the ten items of the System Usability Scale (SUS) [53] (see Figures A3 and A4). The items were translated to German and the wording was simplified.

In the following questions, please rate how well using the glasses worked for you.

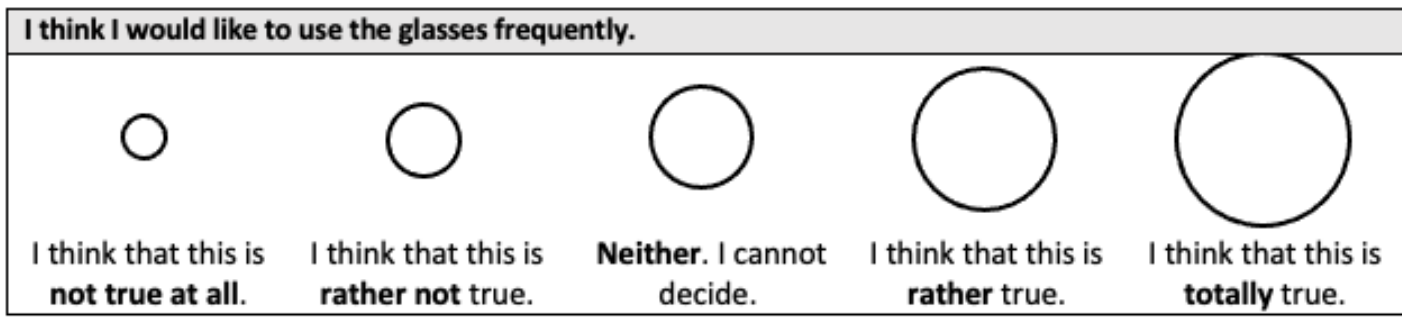

I found the glasses to be more complicated than necessary.
I think that this is I think that this is Neither. I cannot I think that this is I think that this is
not true at all. rather not true.
rather true.

I think the glasses are easy to use.
I think that this is I think that this is Neither. I cannot I think that this is I think that this is
not true at all. rather not true.
rather true.

I think I would need the help of an adult to use the glasses.
$\begin{aligned} & \text { I think that this is I think that this is Neither. I cannot I think that this is I think that this is } \\ & \text { not true at all. rather not true. } \\ & \text { rather true. }\end{aligned}$

\begin{tabular}{|l}
\hline I think the different features of the glasses worked well together. \\
$\begin{array}{l}\text { I think that this is I think that this is Neither. I cannot I think that this is I think that this is } \\
\text { not true at all. rather not true. } \\
\text { rather true. }\end{array}$
\end{tabular}

Figure A3. Questionnaire for device usability assessment (page 1). 

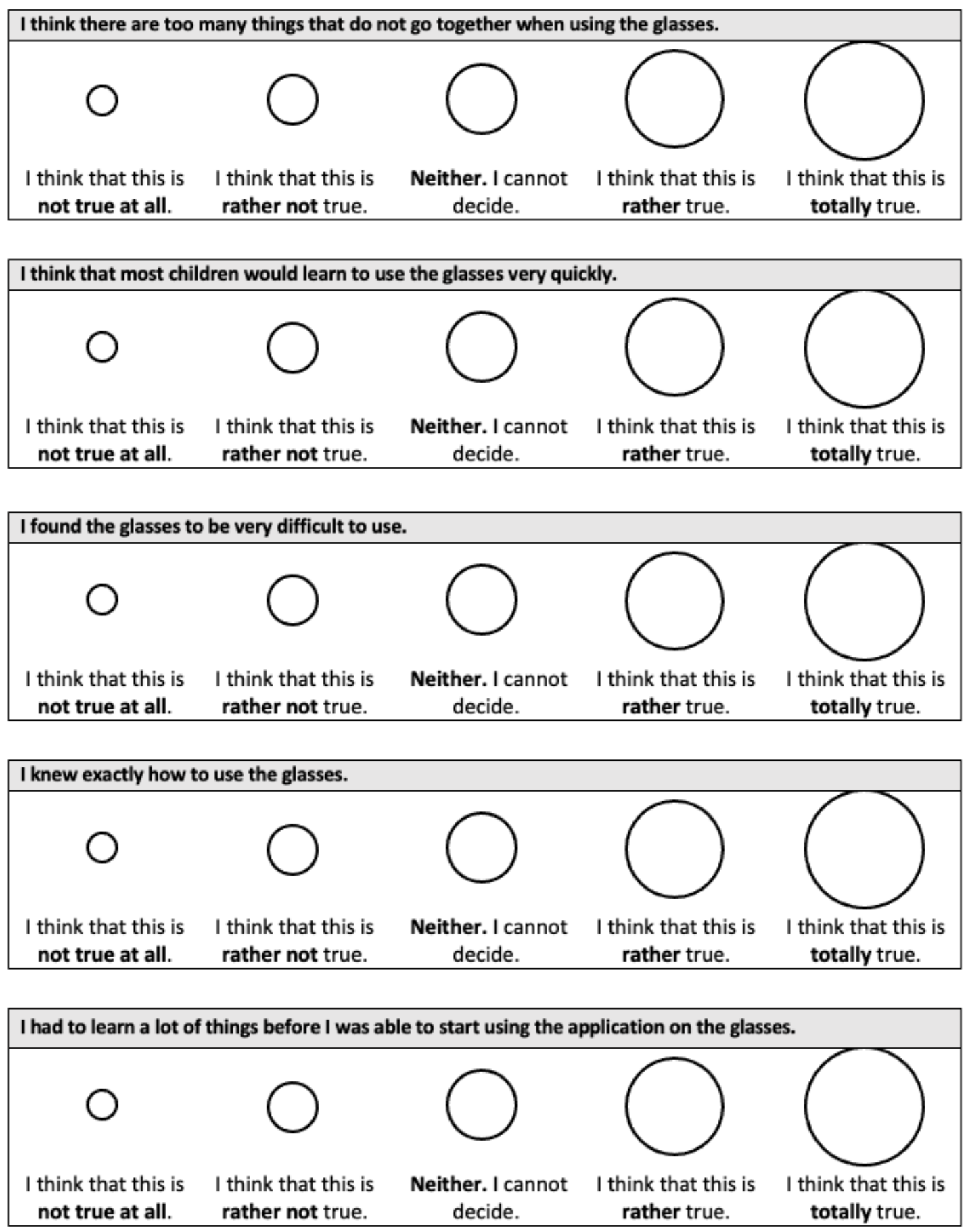

Figure A4. Questionnaire for device usability assessment (page 2).

\section{References}

1. Azuma, R.; Baillot, Y.; Behringer, R.; Feiner, S.; Julier, S.; MacIntyre, B. Recent advances in augmented reality. IEEE Comput. Graph. Appl. 2001, 21, 34-47. [CrossRef]

2. Cheng, K.-H.; Tsai, C.-C. Affordances of augmented reality in science learning: Suggestions for future research. J. Sci. Educ. Technol. 2013, 22, 449-462. [CrossRef]

3. Milgram, P.; Kishino, F. A taxonomy of mixed reality visual displays. IEICE Trans. Inf. Syst. 1994, 77, 1321-1329.

4. Akçayır, M.; Akçayır, G. Advantages and challenges associated with augmented reality for education: A systematic review of the literature. Educ. Res. Rev. 2017, 20, 1-11. [CrossRef] 
5. Garzón, J.; Acevedo, J. Meta-analysis of the impact of augmented reality on students' learning gains. Educ. Res. Rev. 2019, 27, 244-260. [CrossRef]

6. Garzón, J.; Kinshuk; Baldiris, S.; Gutiérrez, J.; Pavón, J. How do pedagogical approaches affect the impact of augmented reality on education? A meta-analysis and research synthesis. Educ. Res. Rev. 2020, 31, 100334. [CrossRef]

7. Ozdemir, M.; Sahin, C.; Arcagok, S.; Demir, M.K. The effect of augmented reality applications in the learning process: A meta-analysis study. Eurasian J. Educ. Res. 2018, 18, 165-186. [CrossRef]

8. Ibáñez, M.-B.; Delgado-Kloos, C. Augmented reality for STEM learning: A systematic review. Comput. Educ. 2018, 123, 109-123. [CrossRef]

9. Lara, O.D.; Labrador, M.A. A survey on human activity recognition using wearable sensors. IEEE Commun. Surv. Tutor. 2013, 15, 1192-1209. [CrossRef]

10. Vrigkas, M.; Nikou, C.; Kakadiaris, I.A. A review of human activity recognition methods. Front. Robot. AI 2015, 2, 28. [CrossRef]

11. Santos, M.E.C.; Chen, A.; Taketomi, T.; Yamamoto, G.; Miyazaki, J.; Kato, H. Augmented reality learning experiences: Survey of prototype design and evaluation. IEEE Trans. Learn. Technol. 2014, 7, 38-56. [CrossRef]

12. Mayer, R. Multimedia Learning, 2nd ed.; Cambridge University Press: New York, NY, USA, 2009.

13. Strzys, M.P.; Thees, M.; Kapp, S.; Kuhn, J. Smartglasses in STEM laboratory courses-The augmented thermal flux experiment. In Proceedings of the Physics Education Research Conference 2018, Washington, DC, USA, 1-2 August 2018. [CrossRef]

14. Thees, M.; Kapp, S.; Strzys, M.P.; Beil, F.; Lukowicz, P.; Kuhn, J. Effects of augmented reality on learning and cognitive load in university physics laboratory courses. Comput. Hum. Behav. 2020, 108, 106316. [CrossRef]

15. Altmeyer, K.; Kapp, S.; Thees, M.; Malone, S.; Kuhn, J.; Brünken, R. The use of augmented reality to foster conceptual knowledge acquisition in STEM laboratory courses-Theoretical background and empirical results. Br. J. Educ. Technol. 2020, 51, 611-628. [CrossRef]

16. Szajna, A.; Stryjski, R.; Woźniak, W.; Chamier-Gliszczyński, N.; Kostrzewski, M. Assessment of augmented reality in manual wiring production process with use of mobile AR glasses. Sensors 2020, 20, 4755. [CrossRef]

17. Ainsworth, S. DeFT: A conceptual framework for considering learning with multiple representations. Learn. Instr. 2006, 16, 183-198. [CrossRef]

18. Evans, G.; Miller, J.; Iglesias Pena, M.; MacAllister, A.; Winer, E. Evaluating the Microsoft HoloLens through an augmented reality assembly application. In Proceedings of the SPIE Defense + Security 2017, Anaheim, CA, USA, 9-13 April 2017; p. 101970V. [CrossRef]

19. Kapp, S.; Thees, M.; Beil, F.; Weatherby, T.; Burde, J.-P.; Wilhelm, T.; Kuhn, J. A comparative study in an undergraduate physics laboratory course. In Proceedings of the CSEDU 2020: 12th International Conference on Computer Supported Education, Prague, Czech Republic, 2-4 May 2020; Volume 2, pp. 197-206. [CrossRef]

20. Wilson, M. Six views of embodied cognition. Psychon. Bull. Rev. 2002, 9, 625-636. [CrossRef] [PubMed]

21. Korbach, A.; Ginns, P.; Brünken, R.; Park, B. Should learners use their hands for learning? Results from an eye-tracking study. J. Comput. Assist. Learn. 2020, 36, 102-113. [CrossRef]

22. Bujak, K.R.; Radu, I.; Catrambone, R.; MacIntyre, B.; Zheng, R.; Golubski, G. A psychological perspective on augmented reality in the mathematics classroom. Comput. Educ. 2013, 68, 536-544. [CrossRef]

23. Yuen, S.C.-Y.; Yaoyuneyong, G.; Johnson, E. Augmented reality: An overview and five directions for AR in education. J. Educ. Technol. Dev. Exch. JETDE 2011, 4, 119-140. [CrossRef]

24. Majeed, Z.H.; Ali, H.A. A review of augmented reality in educational applications. Int. J. Adv. Technol. Eng. Explor. 2020, 7, 20-27. [CrossRef]

25. Dede, C. Immersive interfaces for engagement and learning. Science 2009, 323, 66-69. [CrossRef]

26. Wu, H.-K.; Lee, S.W.-Y.; Chang, H.-Y.; Liang, J.-C. Current status, opportunities and challenges of augmented reality in education. Comput. Educ. 2013, 62, 41-49. [CrossRef]

27. Anderson, C.L.; Anderson, K.M. Wearable technology: Meeting the needs of individuals with disabilities and its applications to education. In Perspectives on Wearable Enhanced Learning (WELL); Buchem, I., Klamma, R., Wild, F., Eds.; Springer: Cham, Switzerland, 2019; pp. 59-77.

28. Barz, M.; Kapp, S.; Kuhn, J.; Sonntag, D. Automatic recognition and augmentation of attended objects in real-time using eye tracking and a head-mounted display. In Proceedings of the ACM Symposium on Eye Tracking Research and Applications, Virtual Event, Germany, 25-27 May 2021; pp. 1-4. [CrossRef]

29. Arici, F.; Yildirim, P.; Caliklar, S..; Yilmaz, R.M. Research trends in the use of augmented reality in science education: Content and bibliometric mapping analysis. Comput. Educ. 2019, 142, 103647. [CrossRef]

30. Kuhn, J.; Lukowicz, P.; Hirth, M.; Weppner, J. GPhysics-Using google glass as experimental tool for wearable-technology enhanced learning in physics. In Proceedings of the 11th International Conference on Intelligent Environments, Prague, Czech Republic, 15-17 July 2015; pp. 212-219. [CrossRef]

31. Zhang, H.; Cui, Y.; Shan, H.; Qu, Z.; Zhang, W.; Tu, L.; Wang, Y. Hotspots and trends of virtual reality, augmented reality and mixed reality in education field. In Proceedings of the 6th International Conference of the Immersive Learning Research Network (iLRN 2020), San Luis Obispo, CA, USA, 21-25 June 2020; pp. 215-219. [CrossRef]

32. Pekrun, R. The control-value theory of achievement emotions: Assumptions, corollaries, and implications for educational research and practice. Educ. Psychol. Rev. 2006, 18, 315-341. [CrossRef] 
33. Pekrun, R.; Goetz, T.; Frenzel, A.C.; Barchfeld, P.; Perry, R.P. Measuring emotions in students' learning and performance: The achievement emotions questionnaire (AEQ). Contemp. Educ. Psychol. 2011, 36, 36-48. [CrossRef]

34. Munoz-Cristobal, J.A.; Jorrin-Abellan, I.M.; Asensio-Perez, J.I.; Martinez-Mones, A.; Prieto, L.P.; Dimitriadis, Y. Supporting teacher orchestration in ubiquitous learning environments: A study in primary education. IEEE Trans. Learn. Technol. 2015, 8, 83-97. [CrossRef]

35. Squire, K.D.; Jan, M. Mad city mystery: Developing scientific argumentation skills with a place-based augmented reality game on handheld computers. J. Sci. Educ. Technol. 2007, 16, 5-29. [CrossRef]

36. Ibanez, M.-B.; de Castro, A.J.; Delgado Kloos, C. An empirical study of the use of an augmented reality simulator in a face-to-face physics course. In Proceedings of the 2017 IEEE 17th International Conference on Advanced Learning Technologies (ICALT), Timisoara, Romania, 3-7 July 2017; pp. 469-471. [CrossRef]

37. Radu, I. Augmented reality in education: A meta-review and cross-media analysis. Pers. Ubiquitous Comput. 2014, 18, 1533-1543. [CrossRef]

38. Nielsen, J. Usability Engineering; Academic Press: San Diego, CA, USA, 1993.

39. Bourges-Waldegg, P.; Moreno, L.; Rojano, T. The role of usability on the implementation and evaluation of educational technology. In Proceedings of the 33rd Annual Hawaii International Conference on System Sciences, Maui, Hawaii, 4-7 January 2000; Volume 1, p. 7. [CrossRef]

40. Papakostas, C.; Troussas, C.; Krouska, A.; Sgouropoulou, C. Measuring user experience, usability and interactivity of a personalized mobile augmented reality training system. Sensors 2021, 21, 3888. [CrossRef] [PubMed]

41. Chang, Y.-L.; Hou, H.-T.; Pan, C.-Y.; Sung, Y.-T.; Chang, K.-E. Apply an augmented reality in a mobile guidance to increase sense of place for heritage places. J. Educ. Technol. Soc. 2015, 18, 166-178.

42. Radu, I.; MacIntyre, B. Using children's developmental psychology to guide augmented-reality design and usability. In Proceedings of the 2012 IEEE International Symposium on Mixed and Augmented Reality (ISMAR), Atlanta, GA, USA, 5-8 November 2012; pp. 227-236. [CrossRef]

43. Munsinger, B.; White, G.; Quarles, J. The usability of the Microsoft HoloLens for an augmented reality game to teach elementary school children. In Proceedings of the 11th International Conference on Virtual Worlds and Games for Serious Applications (VS-Games), Vienna, Austria, 4-6 September 2019; pp. 1-4. [CrossRef]

44. Kennedy, J.; Lemaignan, S.; Montassier, C.; Lavalade, P.; Irfan, B.; Papadopoulos, F.; Senft, E.; Belpaeme, T. Child speech recognition in human-robot interaction: Evaluations and recommendations. In Proceedings of the 2017 ACM/IEEE International Conference on Human-Robot Interaction, Vienna, Austria, 6 March 2017; pp. 82-90. [CrossRef]

45. Oviatt, S.; Cohen, P.R. The paradigm shift to multimodality in contemporary computer interfaces. Synth. Lect. Hum.-Cent. Inform. 2015, 8, 1-243. [CrossRef]

46. Jameson, A.; Kristensson, P.O. Understanding and supporting modality choices. In The Handbook of Multimodal-Multisensor Interfaces: Foundations, User Modeling, and Common Modality Combinations; Oviatt, S., Schuller, B., Cohen, P.R., Sonntag, D., Potamianos, G., Krüger, A., Eds.; Association for Computing Machinery (ACM): New York, NY, USA, 2017; Volume 1, pp. 201-238. [CrossRef]

47. Oviatt, S.; Schuller, B.; Cohen, P.R.; Sonntag, D.; Potamianos, G.; Krüger, A. Introduction: Scope, trends, and paradigm shift in the field of computer interfaces. In The Handbook of Multimodal-Multisensor Interfaces: Foundations, User Modeling, and Common Modality Combinations; Oviatt, S., Schuller, B., Cohen, P.R., Sonntag, D., Potamianos, G., Krüger, A., Eds.; Association for Computing Machinery (ACM): New York, NY, USA, 2017; pp. 1-15. [CrossRef]

48. Southgate, E.; Smith, S.P.; Scevak, J. Asking ethical questions in research using immersive virtual and augmented reality technologies with children and youth. In Proceedings of the 2017 IEEE Virtual Reality (VR), Los Angeles, CA, USA, 18-22 March 2017; pp. 12-18. [CrossRef]

49. Steele, P.; Burleigh, C.; Kroposki, M.; Magabo, M.; Bailey, L. Ethical considerations in designing virtual and augmented reality products-Virtual and augmented reality design with students in mind: Designers' perceptions. J. Educ. Technol. Syst. 2020, 49, 219-238. [CrossRef]

50. Slater, M.; Gonzalez-Liencres, C.; Haggard, P.; Vinkers, C.; Gregory-Clarke, R.; Jelley, S.; Watson, Z.; Breen, G.; Schwarz, R.; Steptoe, W.; et al. The ethics of realism in virtual and augmented reality. Front. Virtual Real. 2020, 1, 1. [CrossRef]

51. Product Safety Warnings and Instructions. Available online: https://support.microsoft.com/en-us/topic/product-safetywarnings-and-instructions-726eab87-f471-4ad8-48e5-9c25f68927ba (accessed on 22 September 2021).

52. Riemer, V.; Schrader, C. Mental model development in multimedia learning: Interrelated effects of emotions and self-monitoring. Front. Psychol. 2019, 10, 899. [CrossRef]

53. Brooke, J. SUS: A “quick and dirty” usability scale. In Usability Evaluation in Industry; Jordan, P.W., Thomas, B., Weerdmeester, B.A., McClelland, I., Eds.; Taylor \& Francis: London, UK, 1996; pp. 189-194.

54. Bruck, S.; Watters, P.A. Estimating cybersickness of simulated motion using the simulator sickness questionnaire (SSQ): A controlled study. In Proceedings of the 2009 Sixth International Conference on Computer Graphics, Imaging and Visualization, Tianjin, China, 11-14 August 2009; pp. 486-488. [CrossRef]

55. Bangor, A.; Kortum, P.; Miller, J. Determining what individual SUS scores mean: Adding an adjective rating scale. J. Usability Stud. 2009, 4, 114-123. 
56. Vovk, A.; Wild, F.; Guest, W.; Kuula, T. Simulator sickness in augmented reality training using the Microsoft HoloLens. In Proceedings of the 2018 CHI Conference on Human Factors in Computing Systems, Montreal, QC, Canada, 21 April 2018; pp. 1-9. [CrossRef]

57. Serio, Á.D.; Ibáñez, M.B.; Kloos, C.D. Impact of an augmented reality system on students' motivation for a visual art course. Comput. Educ. 2013, 68, 586-596. [CrossRef]

58. Sung, E.; Mayer, R.E. When graphics improve liking but not learning from online lessons. Comput. Hum. Behav. 2012, 28, 1618-1625. [CrossRef]

59. Moreno, R.; Mayer, R. Interactive multimodal learning environments. Educ. Psychol. Rev. 2007, 19, 309-326. [CrossRef]

60. Knörzer, L.; Brünken, R.; Park, B. Emotions and multimedia learning: The moderating role of learner characteristics: Emotions in multimedia learning. J. Comput. Assist. Learn. 2016, 32, 618-631. [CrossRef]

61. Parong, J.; Mayer, R.E. Learning science in immersive virtual reality. J. Educ. Psychol. 2018, 110, 785-797. [CrossRef] 